\title{
Overdosing on immediate-release morphine solution has predictable adverse effects
}

\author{
To the Editor:
}

Prescribing responsibility inherently includes adequate ongoing monitoring for medication.

An overdose of morphine can cause respiratory depression; as with any medication, there is a therapeutic window above which toxicities occur. Despite the increasingly widespread use of extended-release morphine for chronic breathlessness when prescribed and monitored in line with international evidence-based recommendations, the literature is not replete with examples of opioid-induced respiratory depression.

Why was the case report by Politis et al. [1] published? Morphine causes drowsiness and obtundation when an overdose is taken. In addition to his $15 \mathrm{mg}$ per $24 \mathrm{~h}$ extended-release morphine, the patient in the case report took 12 times his 4-hourly dose of immediate-release oral morphine solution. The effects he experienced are well-described toxicities on the product information and the patient information sheets in every filled prescription for every opioid. This is not about identifying a new danger; use morphine in an unsafe way and it will be an unsafe drug with predictable side-effects. This letter, therefore, highlights that the generic importance of good prescribing and prescribing responsibility inherently includes adequate ongoing monitoring.

The evidence base for the therapeutic clinical use of opioids in chronic breathlessness has been derived almost entirely from extended-release preparations of morphine [2,3]. This choice of preparation was based on sound pharmacokinetic principles from studies done more than 20 years ago [4] and is borne out by the greater effect size in aggregated data from steady state studies [3]. Even when well with no comorbidities, anyone of us would probably experience toxicity from a statim dose of $30 \mathrm{mg}$ immediate-release morphine solution. The goal of care is to increase the threshold at which the patient becomes breathless during exertion by adequately saturating opioid receptors and to decrease the distress caused by breathlessness in the person's life. This relies on adequate levels of background opioids. The use of as-needed immediate-release oral morphine solution will increase the risk of side-effects because of fluctuating serum levels and increase the potential for overdosing on immediate-release morphine solution.

The approach whereby an immediate-release opioid is used for episodes of breathlessness (usually induced by exertion) has been transferred from pain management without empiric data in breathlessness. However, given the current evidence about chronic breathlessness, it makes no sense to transfer an as-needed immediate-release morphine model that was not even fit for purpose in incident pain. As most episodes of exertion-induced breathlessness resolve with rest within $10 \mathrm{~min}[5,6]$, no opioid preparation with a relevant pharmacokinetic profile has been studied in chronic breathlessness.

Patients need to be monitored as opioids are initiated and during follow-up in a way that is appropriate to the medication being prescribed. In settings where the medication is started during an acute exacerbation, it may be appropriate to review the person within days of discharge to explore whether they needed ongoing morphine.

The basis of the toxicity was that the patient took a much higher than prescribed dose of immediate-release oral morphine solution. When low-dose, extended-release morphine has been used in a steady state, such toxicity has not been reported. Sudden changes in renal function or dehydration due to diarrhoea may put

@ERSpublications

The adverse effects of an overdose of immediate-release morphine in a recent letter to the ERJ are well known http://ow.ly/71D130dMLYd

Cite this article as: Currow DC, Ekström M, Johnson MJ. Overdosing on immediate-release morphine solution has predictable adverse effects. Eur Respir J 2017; 50: 1701091 [https://doi.org/10.1183/ 13993003.01091-2017]. 
even people in a steady state from regular, low-dose, extended-release morphine at risk of harm despite a history of symptomatic benefit. Part of a pharmacist discussing regular, low-dose, extended-release morphine with patients when this medication is initiated is that side-effects may emerge were renal function to worsen.

Chronic breathlessness continues to be a major health problem around the world and its relief a goal of care for millions of people [7]. The most robust evidence base to date is built on the use of regular, low-dose, extended-release morphine. Further research into safety and titration is ongoing. Such work will be crucial to strike the therapeutic balance between maximising symptomatic benefits and minimising any likelihood of harms in these frail people [8].

David C. Currow ${ }^{1,2}$, Magnus Ekström ${ }^{3}$ and Miriam J. Johnson ${ }^{2,4}$

${ }^{1}$ IMPACCT, Faculty of Health, University of Technology Sydney, Ultimo, Australia. ${ }^{2}$ Wolfson Palliative Care Research Centre, Hull York Medical School, University of Hull, Hull, UK. ${ }^{3}$ Dept of Clinical Sciences, Division of Respiratory Medicine and Allergology, Lund University, Lund, Sweden. ${ }^{4}$ Hull York Medical School, Hull University, Hull, UK.

Correspondence: David C. Currow, IMPACCT, Faculty of Health, University of Technology Sydney, New South Wales, 2007, Australia. E-mail: david.currow@uts.edu.au

Received: May 312017 | Accepted: June 092017

Conflict of interest: Disclosures can be found alongside this article at erj.ersjournals.com

\section{References}

1 Politis J, Le B, Smallwood N. Respiratory depression secondary to morphine use in a patient with COPD and refractory breathlessness. Eur Respir J 2017; 49: 1601858.

2 Ekström M, Bajwah S, Bland JM, et al. One evidence base; three stories: do opioids relieve chronic breathlessness? Thorax 2017 [in press https://doi.org/10.1136/thoraxjnl-2016-209868].

3 Ekstrom M, Nilsson F, Abernethy AP, et al. Effects of opioids on breathlessness and exercise capacity in chronic obstructive pulmonary disease. A systematic review. Ann Am Thoracic Soc 2015; 12: 1079-1092.

4 Gourlay GK, Plummer JL, Cherry DA, et al. A comparison of Kapanol (a new sustained-release morphine formulation), MST Continuous, and morphine solution in cancer patients: pharmacokinetic aspects of morphine and morphine metabolites. In: Gebhart GF, Hammond DL, Jensen TS, eds. Proceedings of the 7th World Congress on Pain. Seattle, IASP Press, 1994; pp. 631-643.

5 Maddocks M, Taylor V, Klezlova R, et al. When will I get my breath back? Recovery time of exercise-induced breathlessness in patients with thoracic cancer. Lung Cancer 2012; 76: 128-129.

6 Simon ST, Weingärtner V, Higginson IJ, et al. Definition, categorization, and terminology of episodic breathlessness: consensus by an international Delphi survey. J Pain Symptom Manage 2014; 47: 828-838.

7 Currow DC, Plummer J, Crockett A, et al. A community population survey of prevalence and severity of dyspnea in adults. J Pain Symptom Manage 2009; 38: 533-545.

8 Currow DC, Watts GJ, Johnson MJ, et al. A pragmatic, phase III, multisite, double-blind, placebo-controlled, parallel-arm, dose increment randomised trial of regular, low-dose extended-release morphine for chronic breathlessness: Breathlessness Exertion And Morphine Sulphate (BEAMS) study protocol. BMJ Open 2017; 7: e018100.

\section{Predictable adverse events such as respiratory depression highlight the need for caution when prescribing morphine for refractory breathlessness}

\footnotetext{
From the authors:

"A scientist's aim in a discussion with his colleagues is not to persuade, but to clarify" [1]. We therefore thank D.C. Currow and co-workers for both their comments and the opportunity to continue the discussion regarding the risks and benefits of using opioids to treat refractory chronic breathlessness. The purpose of our recent case report [2] is not to persuade clinicians to prescribe or not prescribe opioids for the off-licence indication of refractory breathlessness, but to highlight and clarify risks of prescribing opioids in this clinical setting and when practising real life medicine, which is so different from the carefully controlled and managed environment of clinical trials.
} 
We readily acknowledge that respiratory depression is a predictable and unremarkable outcome from a significant opioid overdose. Indeed, many physicians cite the risk of respiratory depression as one of their main concerns when considering prescribing opioids for refractory breathlessness [3-7], therefore this event, as rightly noted, is not a new danger. However, the purpose of case reports is to provide detailed and accurate information about individual patients, which is lost and cannot be reported in larger clinical trials. This detailed description of a clinician's experience is essential and allows for discussion as the evidence from clinical trials is translated into the practice of real world medicine.

Therefore, to clarify further this particular case report aimed to discuss not predictable adverse events from an opioid overdose, but: 1) the risks of first initiating opioids for severe breathlessness to patients with acute exacerbations of chronic obstructive pulmonary disease (COPD), as to date these patients have been excluded from all clinical trials investigating the role of opioids for refractory breathlessness; 2) the risks of inadvertent patient dosing errors occurring in COPD patients who are often older, frail, socially isolated and have multiple medical issues; and 3) the need for patient education regarding dosing and adverse events, as well as close supervision and early outpatient review, with ongoing follow-up to monitor treatment response, compliance, side-effects and possible risks [2].

As discussed in our case report, there is currently no evidence to support a regimen that uses both extended release and immediate release morphine preparations together for treating refractory breathlessness [2]. However, it should be noted that the current evidence base for using opioids to treat refractory breathlessness is very limited [8]. Nonetheless, this particular dosing regimen was recommended by the specialist palliative care team to the patient in the case report, because the patient appeared to be actively dying from respiratory failure and had severe and extremely distressing breathlessness at rest (not just on exertion). The case patient required both types of morphine preparation to achieve adequate symptom palliation. Therefore, it is unclear how the comments of D.C. Currow and co-workers regarding the pharmocokinetics of immediate release morphine and its role in managing exertion induced breathlessness bear any relevance to this case report.

Additionally, focusing on the morphine regimen prescribed to the case patient distracts from the fundamental issue, which was that opioid induced respiratory depression occurred after an inadvertent patient dosing error. Such errors may occur with either tablet or liquid medications, therefore the patient could potentially have accidentally overdosed on either an extended release or immediate release morphine preparation. Potential options to prevent patient dosing errors include ceasing morphine prescription on hospital discharge or prescribing daily, extended release morphine administered from a pharmacist-prepared, scheduled, medication dosette box.

We completely agree with D.C. Currow and co-workers that responsible prescribing of opioids inherently includes adequate monitoring and supervision, and indeed we discussed this issue within the case report [2]. There is no doubt that when patients are discharged home with a new prescription of morphine for breathlessness, they should ideally be seen by the prescribing clinician or team within 1-2 weeks of discharge. However, access to urgent outpatient review is often challenging due to high service demands and the ability of each health system to meet those needs. Similarly, for geographically vast countries like Australia, patients may have to travel long distances (requiring a drive of several hours) to attend a hospital outpatient appointment. This poses a significant burden on a patient with severe respiratory disease, who is recovering from an exacerbation and who may be reluctant to return within a few days and wait (potentially hours) to be reviewed. As our aim must be to offer high quality, safe, patient focused care, these issues highlight the absolute need for clinicians to work closely and communicate well with community general practitioners to ensure local, follow-up, supervision and support is immediately available in addition to hospital follow up.

The limited current evidence base suggests that morphine has a small, but important, short term role as an off-licence treatment for chronic refractory breathlessness in stable outpatients [8]. However, our case report highlights that translating limited evidence into wider clinical practice is not straightforward. While we need further clinical trials to better understand optimal morphine dosing and regimen, long term utility and safety, and effect on quality of life, we also need long term, cohort data describing real world

@ERSpublications

Caution is needed when prescribing morphine for refractory breathlessness in the real world http://ow. ly/DPxD30eabIJ

Cite this article as: Smallwood N, Politis J, Le B. Predictable adverse events such as respiratory depression highlight the need for caution when prescribing morphine for refractory breathlessness. Eur Respir J 2017; 50: 1701357 [https://doi.org/10.1183/13993003.01357-2017]. 
experience using opioids in patients with chronic respiratory disease and refractory breathlessness. Finally, as clinicians we must continue to discuss and share our clinical experiences so that we can identify risks, find solutions and prevent avoidable adverse events.

Natasha Smallwood ${ }^{1}$, John Politis ${ }^{1}$ and Brian $\mathrm{Le}^{2}$

${ }^{1}$ Department of Respiratory Medicine, The Royal Melbourne Hospital, Melbourne, Australia. ${ }^{2}$ Palliative and Supportive Care, The Royal Melbourne Hospital, Melbourne, Australia.

Correspondence: Natasha Smallwood, Dept of Respiratory Medicine, The Royal Melbourne Hospital, 300 Grattan St, Parkville, VIC 3050, Australia. E-mail: Natasha.smallwood@mh.org.au

Received: July 062017 | Accepted: July 082017

Conflict of interest: None declared.

\section{References}

1 Szilard L. Close-up: I'm looking for a market for wisdom. LIFE Magazine 1961; 51: 75.

2 Politis J, Le B, Smallwood N. Respiratory depression secondary to morphine use in a patient with COPD and refractory breathlessness. Eur Respir J 2017; 49: 1601858.

3 Hadjiphilippou S, Odogwu SE, Dand P. Doctors' attitudes towards prescribing opioids for refractory dyspnoea: a single-centred study. BMJ Support Palliat Care 2014; 4: 190-192.

4 Janssen DJ, de Hosson SM, bij de Vaate E, et al. Attitudes toward opioids for refractory dyspnea in COPD among Dutch chest physicians. Chron Respir Dis 2015; 12: 85-92.

5 Rocker GM, Young J, Horton R. Using opioids to treat dyspnea in advanced COPD: a survey of Canadian clinicians. Chest 2008; 134: Suppl. 2, s29001.

6 Smallwood N, Gaffney N, Gorelik A, et al. Junior doctors' attitudes to opioids for refractory breathlessness in patients with advanced chronic obstructive pulmonary disease. Intern Med J 2017; in press [https://doi.org/10.1111/ imj.13521].

7 Young J, Donahue M, Farquhar M, et al. Using opioids to treat dyspnea in advanced COPD: attitudes and experiences of family physicians and respiratory therapists. Can Fam Physician 2012; 58: e401-e407.

8 Barnes H, McDonald J, Smallwood N, et al. Opioids for the palliation of refractory breathlessness in adults with advanced disease and terminal illness. Cochrane Database Syst Rev 2016; 3: CD011008. 